\title{
Analysis of Some Software Reliability Growth Models with Learning Effects
}

\author{
Javaid Iqbal
}

Department of Computer Sciences, University of Kashmir, Srinagar, 190006, India

\begin{abstract}
A newly developed software system before its deployment is subjected to vigorous testing so as to minimize the probability of occurrence of failure very soon. Software solutions for safety critical and mission-critical application areas need a much focused level of testing. The testing process is basically carried out to build confidence in the software for its use in real world applications. Thus, reliability of systems is always a matter of concern for us. As we keep on performing the error detection and correction process on our software, the reliability of the system grows. In order to model this growth in the system reliability, many formulations in Software Reliability Growth Models (SRGMs) have been proposed including some based on NonHomogeneous Poisson Process (NHPP). The role of human learning and experiential pattern gains are being studied and incorporated in such models. The realistic assumptions about human learning behavior and experiential gains of new skill-sets for better detection and correction of faults on software are being incorporated and studied in such models. In this paper, a detailed analysis of some select SRGMs with learning effects is presented based on use of seven data sets. The estimation of parameters and comparative analysis based on goodness of fit using seven data sets are presented. Moreover, model comparisons on the basis of total defects predicted by the select models are also tabulated.
\end{abstract}

Index Terms: Software Reliability, Software Reliability Growth Model (SRGM), Non-Homogeneous Poisson Process (NHPP), Learning effect, two-type learning effect.

(C) 2016 Published by MECS Publisher. Selection and/or peer review under responsibility of the Research Association of Modern Education and Computer Science

\section{Introduction}

Many software reliability growth models (SRGMs) under the analytical framework of a Non-Homogeneous Poisson Process (NHPP) have been proposed which aim to better model the error-detection and correction processes by trying to incorporate some realistic underlying assumptions. Goel and Okumoto in [1] proposed an exponential SRGM. Yamada and Ohba in [2] proposed delayed S-shaped SRGM while Ohba in [3]

* Corresponding author.

E-mail address: iamjavaid@gmail.com 
proposed inflection S-shaped SRGM. Gokhale and Trivedi in [4] proposed an enhanced NHPP model which takes into account the time-dependent failures occurring in debugging process. Debugging process was earlier perceived to be perfect and based on the assumption that that each time an error occurred, the fault that caused it can be removed immediately.

Presently, a more realistic assumption is in place for a debugging process of imperfect type and is based on the assumption that the removal of a fault can introduce some new faults [5, 6]. An insight into imperfect debugging can be found in Obha [3,7], Pham[8], Kapur and Younes [9], Shyur [10] and Chiu and Huang [11]. Other realistic assumptions for running environment, testing/debugging strategies and resource allocation can also affect the reliability growth as discussed in Chiu and Huang [11] and Shyur [10]. Many researchers have used NHPP based SRGMs to capture the reliability growth of a software from the processes of testing and debugging [23-28]. Recently, a unified framework for use of SRGMs with learning process and error generation in imperfect debugging environments has been presented in [12]. Chiu and Huang in [11] proposed a learning-effect-based NHPP model that captures the learning effect gained by testing/ debugging staff from inspection and debugging of code. In $[13,14]$ Chiu proposes an improvement model under time-dependent learning effect. In [5] Iqbal, Ahmad and Quadri propose an SRGM that incorporates two types of learning effects and then incorporate a negligence factor also into the SRGM with two types of learning effect in [6]. They basically indicate that the two types of learning effect are autonomous learning and acquired learning with acquired learning gained after a spell of repeated experience/observation of the testing/debugging process by the tester/debugger resulting in concept formation by the tester/debugger about that particular pattern. Recently in $[29,30]$ learning based fault detection rates have been incorporated in imperfect debugging models. In this paper, we refine the definition of autonomous learning as the assimilation of know-how by doing (testing) without role of experience and the acquired learning is refined to the definition of learning that stands acquired after a spell of repeated experience/observation of the testing/debugging process by the tester/debugger.

The rest of the paper is organized as: Section II introduces the non-homogeneous Poisson process. Section III discusses how some select learning based models evolved by improvements starting from the learning model proposed by Chiu and Huang in [11], through improvements by Chiu[13], Iqbal et al [5] introduced the concept of two types of learning in SRGM and later improved it in [6]. This progression in SRGM development is discussed in section III. Section IV discusses parameter estimation. Section V discusses results and presents comparative analysis on the basis of seven data sets listed in the section. This section also presents a comparison of models for total defects predicted using these six out of these seven data Sets. This comparison is presented in six tables. Section VI presents conclusion and is followed by references section. The paper ends with authors' brief profiles.

\section{NHPP Modeling Concepts}

As an error counting process $\{N(t), t \geq 0\}$ with mean $m(t)$ and failure intensity rate $\lambda(t)$ a general NHPP process is written mathematically as:

$$
\operatorname{Pr}(N(t)=k)=\frac{\left[\boldsymbol{m}(\boldsymbol{t})^{\boldsymbol{k}} e^{-\boldsymbol{m}(t)}\right]}{\boldsymbol{k} !}, k=0,1,2,3, \ldots
$$

with mean value function $m(t)$ representing the expected number of errors detected within time $(0, t)$ and mathematically represented as an integral of intensity function between time zero(start) and time t. The conditional software reliability $\mathrm{R}(\mathrm{s} / \mathrm{t})$ which is the probability that no error is detected within a specific time interval $(t, t+s)$, given that an error has occurred at time $t(t \geq 0, s>0)$ and is mathematically written as

$$
R(s / t)=e^{-[m(t+s)-m(t)]}
$$


with limiting value of $R(s / t) \approx 1$ as time approaches to infinity.

\section{Developmental Progression of Some Learning-Based Models}

Here we present a brief account of the progression of development of some select learning based SRGMs.

A. Chiu and Huang Learning Model [11]:

A learning factor $\eta$ that arises from inspection of the testing/debugging codes under the assumption that $\eta$ does not change with time is considered.

Model equation is

$$
f(t)=\frac{d F(t)}{d t}=(\alpha+\eta F(t))(1-F(t))
$$

where autonomous error factor $\boldsymbol{\alpha}>\mathbf{0}$ and learning factor $\boldsymbol{\eta}>0$.

The explicit solution of $\boldsymbol{F}(\boldsymbol{t})$ is given by:

$$
F(t)=1-\frac{1+(\eta / \alpha)}{(\eta / \alpha)+e^{(\alpha+\eta) t}}
$$

and

$$
f(t)=\frac{(\alpha+\eta)^{2} e^{(\alpha+\eta) t}}{\alpha\left((\eta / \alpha)+e^{(\alpha+\eta) t}\right)^{2}}
$$

where mean value function $\boldsymbol{m}(\boldsymbol{t})$ is $\boldsymbol{m}(\boldsymbol{t})=\boldsymbol{a} \boldsymbol{F}(\boldsymbol{t})$

$$
m(t)=a\left\{1-\frac{1+(\eta / \alpha)}{(\eta / \alpha)+e^{(\alpha+\eta) t}}\right\}
$$

intensity function $\lambda(t)=\frac{d(m(t))}{d t}=\boldsymbol{a f}(\boldsymbol{t})$

and error detection rate is

$$
d(t)=\frac{\lambda(t)}{a-m(t)}=(\alpha+\eta)\left(1-\frac{\eta}{\alpha e^{(\alpha+\eta) t}+\eta}\right)
$$

\section{B. Chiu Improvement Model [13,14]:}

A learning factor $\eta$ that arises from inspection of the testing/debugging codes under the assumption that $\eta$ does not change with time and a negligent factor $\tau$, that arises from negligence on part of testers/developers in correcting errors from learnt patterns previously detected, are considered. Model equation is

$$
f(t)=\frac{d F(t)}{d t}=(\alpha+\eta F(t)-\tau)(1-F(t))
$$


The explicit solution of $\boldsymbol{F}(\boldsymbol{t})$ is given by:

$$
F(t)=1-\frac{1+(\eta /(\alpha-\tau))}{(\eta /(\alpha-\tau))+e^{(\alpha+\eta-\tau) t}}
$$

and

$$
f(t)=\frac{(\alpha+\eta)^{2} e^{(\alpha+\eta) t}}{(\alpha-\tau)\left((\eta /(\alpha-\tau))+e^{(\alpha+\eta-\tau) t}\right)^{2}}
$$

Where mean value function $\boldsymbol{m}(\boldsymbol{t})$ is $\boldsymbol{m}(\boldsymbol{t})=\boldsymbol{a} \boldsymbol{F}(\boldsymbol{t})$

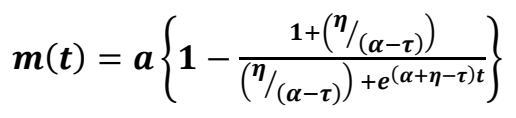

intensity function $\lambda(t)=\frac{d(m(t))}{d t}=\boldsymbol{a} f(t)$ and error detection rate is

$$
d(t)=\frac{\lambda(t)}{a-m(t)}=(\alpha-\tau+\eta)\left(1-\frac{\eta}{\alpha e^{(\alpha+\eta-\tau) t}+\eta}\right)
$$

\section{A Two-Type Learning Model [5]:}

Two type of learning effect, which are autonomous learning $\eta_{1}$ and acquired learning $\eta_{2}$ which represents experiential gains in learning are considered.

Model equation is

$$
f(t)=\frac{d F(t)}{d t}=\left(\eta_{1} \alpha+\eta_{2} F(t)\right)(1-F(t))
$$

where autonomous error factor, $\boldsymbol{\alpha}>\mathbf{0}$, type-I learning factor(autonomous learning) $\boldsymbol{\eta}_{\mathbf{1}}>0$ and type-II learning factor(acquired learning) $\boldsymbol{\eta}_{\mathbf{2}}>0$. The explicit solution of $\boldsymbol{F}(\boldsymbol{t})$ is given by

$$
F(t)=1-\frac{1+\left(\eta_{2} / \eta_{1} \alpha\right)}{\left(\eta_{2} / \eta_{1} \alpha\right)+e^{\left(\eta_{1} \alpha+\eta_{2}\right) t}}
$$

and

$$
f(t)=\frac{\left(\eta_{1} \alpha+\eta_{2}\right)^{2} e^{\left(\eta_{1} \alpha+\eta_{2}\right) t}}{\alpha\left(\left(\eta_{2} / \eta_{1} \alpha\right)+e^{\left(\eta_{1} \alpha+\eta_{2}\right) t}\right)^{2}}
$$

The mean value function $\boldsymbol{m}(\boldsymbol{t})$ is 


$$
m(t)=a F(t)=a\left\{1-\frac{1+\left({ }^{\eta_{2}} / \eta_{1} \alpha\right)}{\left(\eta_{2} / \eta_{1} \alpha\right)+e^{\left(\eta_{1} \alpha+\eta_{2}\right) t}}\right\},
$$

the intensity function is

$$
\lambda(t)=\frac{d(m(t))}{d t}=a f(t)=a\left\{\frac{\left(\eta_{1} \alpha+\eta_{2}\right)^{2} e^{\left(\eta_{1} \alpha+\eta_{2}\right) t}}{\alpha\left(\left(\eta_{2} / \eta_{1} \alpha\right)+e^{\left(\eta_{1} \alpha+\eta_{2}\right) t}\right)^{2}}\right\}
$$

and variation in error detection rate per error at time $\mathbf{t}$ is given by

$$
d(t)=\frac{\lambda(t)}{a-m(t)}=\left(\eta_{1} \alpha+\eta_{2}\right)\left(1-\frac{\eta_{2}}{\eta_{1} \alpha e^{\left(\eta_{1} \alpha+\eta_{2}\right) t}+\eta_{2}}\right)
$$

\section{ATwo-Type Learning Model with negligence factor [6]}

Two types of learning effect, which are autonomous learning $\eta_{1}$ and acquired learning $\eta_{2}$ which represents experiential gains in learning and a negligence factor $\tau$ that arises from the negligence on part of testers/developers in correcting errors from learnt patterns previously detected are considered.

The model equation is:

$$
f(t)=\frac{d F(t)}{d t}=\left(\eta_{1} \alpha+\eta_{2} F(t)-\tau\right)(1-F(t))
$$

where $\boldsymbol{\alpha}>\mathbf{0}, \boldsymbol{\eta}_{\mathbf{1}}>0$ and $\boldsymbol{\eta}_{\mathbf{2}}>0$.

The explicit solution of $\boldsymbol{F}(\boldsymbol{t})$ is given by

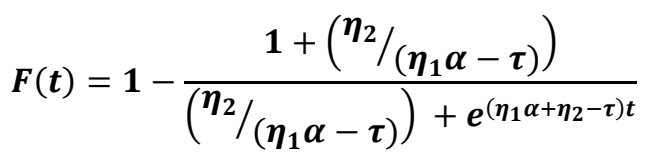

and

$$
f(t)=\frac{\left(\eta_{1} \alpha+\eta_{2}-\tau\right)^{2} e^{\left(\eta_{1} \alpha+\eta_{2}-\tau\right) t}}{\alpha\left(\left(\eta_{2} /\left(\eta_{1} \alpha-\tau\right)\right)+e^{\left(\eta_{1} \alpha+\eta_{2}-\tau\right) t}\right)^{2}}
$$

The mean value function $\boldsymbol{m}(\boldsymbol{t})$ is

$$
m(t)=a F(t)
$$

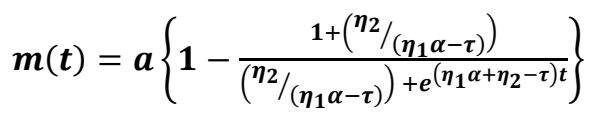


whereas the intensity function 


$$
\lambda(t)=\frac{d(m(t))}{d t}=a f(t)=a\left\{\frac{\left(\eta_{1} \alpha+\eta_{2}-\tau\right)^{2} e^{\left(\eta_{1} \alpha+\eta_{2}-\tau\right) t}}{\alpha\left(\left(\eta_{2} /\left(\eta_{1} \alpha-\tau\right)\right)+e^{\left(\eta_{1} \alpha+\eta_{2}-\tau\right) t}\right)^{2}}\right\}
$$

and variation in error detection rate per error at time is given by

$$
d(t)=\frac{\lambda(t)}{a-m(t)}=\left(\eta_{1} \alpha+\eta_{2}-\tau\right)\left(1-\frac{\eta_{2}}{\left(\eta_{1} \alpha-\tau\right) e^{\left(\eta_{1} \alpha+\eta_{2}-\tau\right) t}+\eta_{2}}\right)
$$

\section{Parameter Estimation}

Fitting the proposed models to the actual data is done by estimating the model parameters. We have used SPSS to estimate the model parameters by using Regression under Non-linear mode. The estimated parameters are presented in different tables to present a comparative analysis for different listed data sets. The mean value functions represented in equations (1) to (4) are used in estimation of parameters.

The following table-1 presents the datasets by labels and presents the sources of data sets listed.

Table 1. Sources of the Datasets

\begin{tabular}{|c|l|l|}
\hline Label & Reference & Dataset \\
\hline$[1]$ & Zhang and Pham[16] & Failure Data of Misra System \\
\hline$[2]$ & Shyur [10] & Failure Data of Misra System \\
\hline$[3]$ & Hossain and Dahiya[17] & Failure Data of NTDS System \\
\hline$[4]$ & Pham and Zhang [18] & Failure data of Tandem Software \\
\hline$[5]$ & Bai, Hu, Xie and Ng [19] & Failure Data of Space program \\
\hline$[6]$ & Pham[20] & Failure data of real time control system \\
\hline$[7]$ & Jeske and Zhang [21] & Failure Data of wireless data service system \\
\hline
\end{tabular}

The following table-2 presents the mean value functions and FDRs of some select models.

Table 2. Model Names and Mean value Function

$$
\begin{aligned}
& \text { Goel Okumotto [1] } \\
& \boldsymbol{m}(\boldsymbol{t})=\boldsymbol{a}\left(1-\boldsymbol{e}^{-\boldsymbol{b} t}\right)
\end{aligned}
$$

Chiu and Huang Learning Model [11]

$m(t)=a\left\{1-\frac{1+(\eta / \alpha)}{(\eta / \alpha)+e^{(\alpha+\eta) t}}\right\}$

2-type Learning Model-1(2TL1) [5]

$$
m(t)=a\left\{1-\frac{1+\left(\eta_{2} / \eta_{1} \alpha\right)}{\left(\eta_{2} / \eta_{1} \alpha\right)+e^{\left(\eta_{1} \alpha+\eta_{2}\right) t}}\right\}
$$

2-type Learning Model-2 (2TL2) [6]

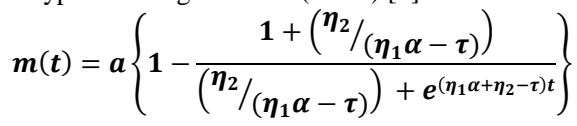

The following table-3 presents the values of parameters of select models using seven data sets which are 
listed in table 1 using mean value functions listed in table 2.

Table 3. Estimation of Parameters under Seven Data Sets for Select Models

\begin{tabular}{|c|c|}
\hline \multicolumn{2}{|c|}{ Comparison under dataset[1] } \\
\hline Model & Parameters \\
\hline $\mathrm{G}-\mathrm{O}[1]$ & $a=135.974, b=.138$ \\
\hline Chiu[11] & $\mathrm{a}=135.965, \alpha=.138, \eta=1.000 \mathrm{E}-4$ \\
\hline 2TL1[5] & $\mathrm{a}=135.965, \alpha=49.216, \eta 1=.003, \eta 2=1.000 \mathrm{E}-4$ \\
\hline 2TL2[6] & $\mathrm{a}=135.974, \alpha=2.611, \eta 1=2.566, \eta 2=.001, \tau=6.562$ \\
\hline \multicolumn{2}{|c|}{ Comparison under dataset[2] } \\
\hline Model & Parameters \\
\hline $\mathrm{G}-\mathrm{O}[1]$ & $a=218.159, b=.041$ \\
\hline Chiu[11] & $\mathrm{a}=215.706, \alpha=.042, \eta=.001$ \\
\hline 2TL1[5] & $\mathrm{a}=215.706, \alpha=56.69, \eta 1=.001, \eta 2=.001$ \\
\hline 2TL2[6] & $\mathrm{a}=210.134, \alpha=.094, \eta 1=.442, \eta 2=1.000 \mathrm{E}-5, \tau=7.007 \mathrm{E}-5$ \\
\hline \multicolumn{2}{|c|}{ Comparison under dataset[3] } \\
\hline Model & Parameters \\
\hline G-O[1] & $\mathrm{a}=33.6, \mathrm{~b}=.063$ \\
\hline Chiu[11] & $\mathrm{a}=24.821, \alpha=.024, \eta=.343$ \\
\hline 2TL1[5] & $\mathrm{a}=24.821, \alpha=.056, \eta 1=.424, \eta 2=.343$ \\
\hline 2TL2[6] & $\mathrm{a}=24.821, \alpha=.217, \eta 1=.658, \eta 2=.343, \tau=.119$ \\
\hline \multicolumn{2}{|c|}{ Comparison under dataset[4] } \\
\hline Model & Parameters \\
\hline G-O[1] & $\mathrm{a}=133.761, \mathrm{~b}=.015$ \\
\hline Chiu[11] & $\mathrm{a}=133.496, \alpha=.146, \eta=.001$ \\
\hline 2TL1[5] & $\mathrm{a}=133.496, \alpha=153.843, \eta 1=.001, \eta 2=.001$ \\
\hline 2TL2[6] & $\mathrm{a}=133.496, \alpha=.002, \eta 1=909.569, \eta 2=.001, \tau=1.748$ \\
\hline \multicolumn{2}{|c|}{ Comparison under dataset[5] } \\
\hline Model & Parameters \\
\hline G-O[1] & $\mathrm{a}=18.257, \mathrm{~b}=.397$ \\
\hline Chiu[11] & $\mathrm{a}=18.254, \alpha=.397, \eta=.001$ \\
\hline $2 \mathrm{TL1}[5]$ & $\mathrm{a}=18.254, \alpha=.049, \eta 1=8.151, \eta 2=.001$ \\
\hline 2TL2[6] & $\mathrm{a}=18.257, \alpha=23.658, \eta 1=.665, \eta 2=1.000 \mathrm{E}-5, \tau=15.341$ \\
\hline \multicolumn{2}{|c|}{ Comparison under dataset[6] } \\
\hline Model & Parameters \\
\hline $\mathrm{G}-\mathrm{O}[1]$ & $\mathrm{a}=124.44, \mathrm{~b}=.051$ \\
\hline Chiu[11] & $\mathrm{a}=124.171, \alpha=.051, \eta=.001$ \\
\hline 2TL1[5] & $\mathrm{a}=124.171, \alpha=.001, \eta 1=88.486, \eta 2=.001$ \\
\hline 2TL2[6] & $\mathrm{a}=124.437, \alpha=.163, \eta 1=5.874, \eta 2=1.000 \mathrm{E}-5, \tau=.909$ \\
\hline \multicolumn{2}{|c|}{ Comparison under dataset[7] } \\
\hline Model & Parameters \\
\hline G-O[1] & $\mathrm{a}=23.092, \mathrm{~b}=.559$ \\
\hline Chiu[11] & $\mathrm{a}=22.252, \alpha=.493, \eta=.332$ \\
\hline 2TL1[5] & $\mathrm{a}=22.252, \alpha=.211, \eta 1=.2 .338, \eta 2=.332$ \\
\hline 2TL2[6] & $\mathrm{a}=22.252, \alpha=9.048, \eta 1=.195, \eta 2=.332, \tau=1.272$ \\
\hline
\end{tabular}

\section{Results and Comparative Analysis}

There are many comparison criteria as defined in [22] wherein the authors have presented analysis and ranking of software reliability models based on weighted criteria. The comparison criteria used is $R^{2}$ measure also called as coefficient of multiple determinations $\left(R^{2}\right)$ which is usually used to depict the goodness-of-fit and is expressible as: [15]

$$
R^{2}=1-\frac{(\text { ResidualSumofSquares })}{(\text { CorrectedSumofSquares })}
$$




$$
R^{2}=1-\frac{\sum_{i=1}^{n}\left(m_{i}-m\left(t_{i}\right)\right)^{2}}{\sum_{i=1}^{n}\left(m_{i}-\sum_{k=1}^{n} m_{k} / n\right)^{2}}
$$

$\boldsymbol{R}^{2}$ represents a measure of the percentage of the total variation about the mean for the fitted curve. It lies in the range of 0 to 1 , with a larger $\boldsymbol{R}^{2}$ value indicating a better representation of variation about the mean of the data set by the model equation. However, a smaller $\boldsymbol{R}^{2}$ value indicates that the model equation fails to represent the variations in the data set. Obviously, a near-one value of $\boldsymbol{R}^{\mathbf{2}}$ is highly desirable [15]. A comparative analysis of some select models using $\boldsymbol{R}^{2}$ measure is presented using seven datasets and model comparisons on the basis of total defects predicted by the select models are also tabulated.

The following table-4 presents the results of goodness-of-fit under $R^{2}$ comparison criteria

Table 4. Goodness-of-fit under R2comparison Criteria

\begin{tabular}{|c|c|c|c|c|}
\hline \multicolumn{5}{|c|}{ Comparison under R-sq for given datasets } \\
\hline Dataset & G-O [1] & Chiu[11] & 2TL1[5] & 2TL2 [6] \\
\hline$[1]$ & .966 & .966 & .966 & .966 \\
\hline$[2]$ & .989 & .989 & .989 & .989 \\
\hline$[3]$ & .919 & .992 & .992 & .992 \\
\hline$[4]$ & .990 & .990 & .990 & .990 \\
\hline$[5]$ & .934 & .934 & .934 & .934 \\
\hline$[6]$ & .978 & .977 & .977 & .978 \\
\hline$[7]$ & .987 & .989 & .989 & .989 \\
\hline
\end{tabular}

The following tables 5-11 present the comparison of models for total defects predicted under listed data sets

Table 5. Comparison of Models for Total Defects Predicted using Data Set [1]

$\begin{array}{ccccc}\text { Defects } & \text { GO }[1] & \text { Chiu[11] } & 2 \text { TL1[5] } & 2 \text { TL2[6] } \\ 27 & 17.55773 & 17.55748 & 17.55509 & 17.53115 \\ 43 & 32.84831 & 32.84799 & 32.8448 & 32.81288 \\ 54 & 46.1645 & 46.1642 & 46.16116 & 46.13079 \\ 64 & 57.76122 & 57.76101 & 57.75864 & 57.73498 \\ 75 & 67.86052 & 67.86041 & 67.85894 & 67.84422 \\ 82 & 76.65575 & 76.65574 & 76.6552 & 76.6498 \\ 84 & 84.31529 & 84.31537 & 84.31568 & 84.31883 \\ 89 & 90.98579 & 90.98595 & 90.98697 & 90.99726 \\ 92 & 96.79496 & 96.79517 & 96.79674 & 96.81247 \\ 93 & 101.854 & 101.8543 & 101.8562 & 101.8756 \\ 97 & 106.2598 & 106.2601 & 106.2622 & 106.2836 \\ 104 & 110.0967 & 110.097 & 110.0992 & 110.1209 \\ 106 & 113.4382 & 113.4384 & 113.4405 & 113.4613 \\ 111 & 116.3482 & 116.3484 & 116.3503 & 116.369 \\ 116 & 118.8824 & 118.8826 & 118.8842 & 118.8999 \\ 122 & 121.0895 & 121.0896 & 121.0908 & 121.1028 \\ 122 & 123.0115 & 123.0116 & 123.0123 & 123.0201 \\ 127 & 124.6853 & 124.6853 & 124.6857 & 124.6887 \\ 128 & 126.143 & 126.143 & 126.1428 & 126.141 \\ 129 & 127.4125 & 127.4124 & 127.4117 & 127.4049 \\ 131 & 128.5181 & 128.5179 & 128.5167 & 128.5048 \\ 132 & 129.4809 & 129.4807 & 129.479 & 129.462 \\ 134 & 130.3194 & 130.3191 & 130.3169 & 130.2951 \\ 135 & 131.0496 & 131.0493 & 131.0466 & 131.02 \\ 136 & 131.6855 & 131.6851 & 131.682 & 131.6509\end{array}$


Table 6.Comparison of models for Total Defects Predicted using Data Set [2]

$\begin{array}{ccccc}\text { Defects } & \text { GO[1] } & \text { Chiu[11] } & \text { 2TL1[5] } & \text { 2TL2[6] } \\ 13 & 9.389554 & 9.377357 & 9.377357 & 5.560235 \\ 20 & 12.8108 & 12.79549 & 12.79549 & 9.389434 \\ 26 & 18.49874 & 18.47969 & 18.47969 & 12.81065 \\ 31 & 23.54737 & 23.52641 & 23.52641 & 18.49855 \\ 34 & 28.78153 & 28.75984 & 28.75984 & 23.54717 \\ 36 & 34.40701 & 34.38584 & 34.38584 & 28.78131 \\ 41 & 39.90221 & 39.88266 & 39.88266 & 34.4068 \\ 45 & 46.54956 & 46.53319 & 46.53319 & 39.90202 \\ 47 & 51.53715 & 51.52387 & 51.52387 & 46.54939 \\ 51 & 55.91801 & 55.9078 & 55.9078 & 51.53702 \\ 58 & 60.19674 & 60.18975 & 60.18975 & 55.91791 \\ 58 & 62.52843 & 62.52325 & 62.52325 & 60.19667 \\ 63 & 65.9616 & 65.9591 & 65.9591 & 62.52838 \\ 66 & 68.42484 & 68.42425 & 68.42425 & 65.96157 \\ 69 & 72.57233 & 72.57486 & 72.57486 & 68.42483 \\ 72 & 76.19502 & 76.20011 & 76.20011 & 72.57235 \\ 76 & 79.81904 & 79.82643 & 79.82643 & 76.19507 \\ 86 & 85.34595 & 85.35619 & 85.35619 & 79.81911 \\ 89 & 86.71047 & 86.72127 & 86.72127 & 85.34605 \\ 90 & 87.36045 & 87.37149 & 87.37149 & 86.71058 \\ 92 & 90.29866 & 90.31055 & 90.31055 & 87.36056 \\ 96 & 92.8607 & 92.87302 & 92.87302 & 90.29878 \\ 101 & 96.12987 & 96.14225 & 96.14225 & 92.86082 \\ 101 & 97.43348 & 97.44571 & 97.44571 & 96.12999\end{array}$

Table 7. Comparison of Models for Total Defects Predicted using Data Set [3]

$\begin{array}{ccccc}\text { Defects } & \text { GO[1] } & \text { Chiu[11] } & \text { 2TL1[5] } & \text { 2TL2[6] } \\ 1 & 1.851063 & 0.616585 & 0.616584 & 0.616585 \\ 2 & 4.161494 & 1.743671 & 1.743667 & 1.743671 \\ 3 & 6.131381 & 3.151695 & 3.151688 & 3.151694 \\ 4 & 6.814544 & 3.763111 & 3.763104 & 3.76311 \\ 5 & 7.969451 & 4.964748 & 4.96474 & 4.964748 \\ 6 & 8.290182 & 5.338404 & 5.338395 & 5.338403 \\ 7 & 9.07456 & 6.328614 & 6.328605 & 6.328613 \\ 8 & 10.27932 & 8.061859 & 8.061851 & 8.061859 \\ 9 & 11.00205 & 9.219136 & 9.219129 & 9.219136 \\ 10 & 11.9764 & 10.8984 & 10.89839 & 10.8984 \\ 11 & 12.11212 & 11.1413 & 11.14129 & 11.1413 \\ 12 & 12.90874 & 12.59865 & 12.59865 & 12.59865 \\ 13 & 13.03861 & 12.84003 & 12.84002 & 12.84003 \\ 14 & 14.17135 & 14.95556 & 14.95555 & 14.95556 \\ 15 & 14.65455 & 15.8461 & 15.8461 & 15.8461 \\ 16 & 14.77346 & 16.06253 & 16.06253 & 16.06253 \\ 17 & 15.12573 & 16.69539 & 16.69539 & 16.69539 \\ 18 & 15.47142 & 17.30184 & 17.30184 & 17.30184 \\ 19 & 16.14349 & 18.42877 & 18.42877 & 18.42877 \\ 20 & 16.25306 & 18.60495 & 18.60495 & 18.60495 \\ 21 & 17.41382 & 20.3175 & 20.3175 & 20.3175 \\ 22 & 20.45052 & 23.29559 & 23.29559 & 23.29559 \\ 23 & 21.01748 & 23.62514 & 23.62514 & 23.62514 \\ 24 & 26.5052 & 24.77606 & 24.77605 & 24.77606 \\ 25 & 26.59398 & 24.7792 & 24.77919 & 24.7792\end{array}$


Table 8. Comparison of Models for Total Defects Predicted using Data Set [4]

$\begin{array}{ccccc}\text { Defects } & \text { GO[1] } & \text { Chiu[11] } & \text { 2TL1[5] } & \text { 2TL2[6] } \\ 16 & 9.770512 & 9.763303 & 9.763301 & 9.763301 \\ 24 & 17.64551 & 17.6352 & 17.6352 & 17.6352 \\ 27 & 25.22674 & 25.21546 & 25.21546 & 25.21546 \\ 33 & 32.32782 & 32.31718 & 32.31717 & 32.31717 \\ 41 & 40.80269 & 40.79441 & 40.7944 & 40.7944 \\ 49 & 48.20764 & 48.20246 & 48.20246 & 48.20246 \\ 54 & 55.01122 & 55.00938 & 55.00937 & 55.00937 \\ 58 & 63.66981 & 63.67229 & 63.67229 & 63.67229 \\ 69 & 71.36726 & 71.37296 & 71.37295 & 71.37295 \\ 75 & 76.64714 & 76.65427 & 76.65426 & 76.65426 \\ 81 & 82.3215 & 82.32902 & 82.32902 & 82.32902 \\ 86 & 86.25277 & 86.25962 & 86.25962 & 86.25962 \\ 90 & 88.97659 & 88.98241 & 88.98241 & 88.98241 \\ 93 & 91.26569 & 91.27022 & 91.27023 & 91.27023 \\ 96 & 93.43779 & 93.44069 & 93.4407 & 93.4407 \\ 98 & 95.49886 & 95.49981 & 95.49982 & 95.49982 \\ 99 & 97.45458 & 97.45328 & 97.45328 & 97.45328 \\ 100 & 99.31033 & 99.30648 & 99.30649 & 99.30649 \\ 100 & 101.0712 & 101.0646 & 101.0646 & 101.0646 \\ 100 & 102.7421 & 102.7324 & 102.7324 & 102.7324\end{array}$

Table 9. Comparison of Models for Total Defects Predicted using Data Set [5]

$\begin{array}{ccccc}\text { Defects } & \text { GO[1] } & \text { Chiu[11] } & 2 \text { TL1[5] } & \text { 2TL2[6] } \\ 1 & 0.144261 & 0.144189 & 0.144189 & 0.144262 \\ 2 & 0.429373 & 0.429168 & 0.429166 & 0.429375 \\ 3 & 1.054572 & 1.05412 & 1.054115 & 1.054578 \\ 4 & 1.854199 & 1.853515 & 1.853508 & 1.85421 \\ 5 & 2.740242 & 2.739408 & 2.739397 & 2.740259 \\ 6 & 3.752057 & 3.75118 & 3.751166 & 3.752081 \\ 7 & 5.1214 & 5.120669 & 5.120651 & 5.121434 \\ 8 & 6.733016 & 6.732716 & 6.732696 & 6.733063 \\ 9 & 8.266495 & 8.266813 & 8.266793 & 8.266553 \\ 10 & 9.998512 & 9.999685 & 9.999666 & 9.998582 \\ 11 & 11.64348 & 11.6455 & 11.64548 & 11.64356 \\ 12 & 13.00265 & 13.00525 & 13.00524 & 13.00272 \\ 13 & 14.37015 & 14.37307 & 14.37307 & 14.37022 \\ 14 & 15.72448 & 15.72717 & 15.72718 & 15.72453 \\ 15 & 16.7745 & 16.77626 & 16.77629 & 16.77452 \\ 16 & 17.44575 & 17.44627 & 17.4463 & 17.44574 \\ 17 & 17.9312 & 17.93013 & 17.93018 & 17.93116 \\ 18 & 18.15346 & 18.1512 & 18.15126 & 18.1534 \\ 19 & 18.2279 & 18.22505 & 18.22511 & 18.22783 \\ 20 & 18.24948 & 18.2464 & 18.24646 & 18.2494 \\ 21 & 18.25548 & 18.25232 & 18.25238 & 18.2554\end{array}$

Table-10: Comparison of models for total defects predicted using data Set [6]:

We skip analysis for the lengthy real time control data set. However $\mathrm{R}^{2}$ analysis is presented for this dataset also. 
Table 10. Comparison of Models for Total Defects Predicted using Data Set [7]

$\begin{array}{ccccc}\text { Defects } & \text { GO[1] } & \text { Chiu[11] } & 2 \text { TL1[5] } & 2 \text { TL2[6] } \\ 1 & 1.66715 & 1.451695 & 1.451693 & 1.451695 \\ 2 & 2.843703 & 2.52046 & 2.520456 & 2.52046 \\ 4 & 3.955647 & 3.56411 & 3.564105 & 3.56411 \\ 5 & 4.305553 & 3.899213 & 3.899208 & 3.899213 \\ 6 & 6.929299 & 6.510605 & 6.510599 & 6.510605 \\ 7 & 7.233704 & 6.824362 & 6.824356 & 6.824362 \\ 9 & 8.378618 & 8.022904 & 8.022898 & 8.022904 \\ 10 & 10.67193 & 10.50022 & 10.50022 & 10.50022 \\ 11 & 12.42447 & 12.44267 & 12.44266 & 12.44267 \\ 12 & 13.20017 & 13.30905 & 13.30905 & 13.30905 \\ 14 & 13.38647 & 13.51728 & 13.51727 & 13.51728 \\ 15 & 13.74862 & 13.92189 & 13.92189 & 13.92189 \\ 16 & 15.92825 & 16.33299 & 16.33299 & 16.33299 \\ 18 & 16.47513 & 16.92416 & 16.92416 & 16.92416 \\ 20 & 19.45476 & 19.91598 & 19.91598 & 19.91598 \\ 21 & 21.69196 & 21.66205 & 21.66205 & 21.66205 \\ 22 & 21.74798 & 21.6962 & 21.6962 & 21.6962 \\ 22 & 21.9273 & 21.80123 & 21.80123 & 21.80123 \\ 22 & 22.46186 & 22.06916 & 22.06916 & 22.06916\end{array}$

\section{Conclusion}

In this paper, a detailed analysis of some select SRGMs with learning effects is presented, the developmental progression is shown. Seven data sets have been used for this detailed analysis and parameter estimation is also presented based on seven data sets. The parameters estimated are competing with other famous models. $R^{2}$ comparison criteria shows fairly good values to validate the models. Finally, model comparisons on the basis of total defects predicted by the select models are also presented in tables.

\section{References}

[1] A. L. Goel and K. Okumoto, "Time-dependent error-detection rate model for software and other performance measures,” IEEE Transactions on Reliability, vol. 28, pp. 206-211, 1979.

[2] S. Yamada and H. Ohba "S-shaped software reliability modeling for software error detection", 1983, IEEE Trans Reliab; 32:475-84.

[3] M. Ohba, "Inflexion S-shaped software reliability growth models", Stochastic Models in Reliability Theory (S. Osaki, Y. Hatoyama, Eds), 1984, pp 144 - 162; Springer- Verlag Merlin.

[4] S.S. Gokhale, and K.S.Trivedi "A time/structure based software reliability model" Ann Software Eng; 1999, 8:85-121.

[5] J. Iqbal, N. Ahmad, and S.M.K. Quadri, "A Software Reliability Growth Model with Two types of Learning", Proceedings of the 1st IEEE International Conference on Machine Intelligence Research and Advancement, SMVDU, Jammu, India, pp. 498-503, 2013.

[6] J. Iqbal, N. Ahmad, and S.M.K. Quadri, "A software reliability growth model with two types of learning and a negligence factor," Image Information Processing (ICIIP), 2013 IEEE Second International Conference on , vol., no., pp.678,683, 9-11 Dec. 2013.

[7] M. Ohba, "Software reliability analysis models," IBM Journal of Research Development, vol. 28, pp. 428-443, 1984.

[8] H. Pham, "Software reliability assessment: Imperfect debugging and multiple failure types in software development”, 1993; EG\&G-RAAM-10737, Idaho National Engineering Laboratory. 
[9] P.K. Kapur, and S. Younes, "Modeling an imperfect debugging phenomenon in software reliability", Microelectronics and Reliability, 1996, Vol. 36, pp. 645-50.

[10] H.J. Shyur, "A stochastic software reliability model with imperfect debugging and change-point", J Syst Software, 2003, 66:135-41.

[11] K.C. Chiu, Y.S. Huang, and T.Z. Lee, "A study of software reliability growth from the perspective of learning effects", Reliability Engineering and System Safety, 2008, 93: 1410-1421.

[12] P. K. Kapur, H. Pham, S. Anand, K. Yadav, "A unified approach for developing software reliability growth models in the presence of imperfect debugging and error generation", IEEE Transactions on Reliability 60 (1) , 331-340, 2011.

[13] K.C. Chiu, "An improved model of software reliability growth under time-dependent learning effects," Quality and Reliability (ICQR), 2011 IEEE International Conference on, vol., no., pp.191, 194, 14-17 Sept. 2011.

[14] K.C. Chiu, "A discussion of software reliability growth models with time-varying learning effects", American Journal of Software engineering and applications. Vol. 2, No. 3, 2013, pp 92-104. doi: 10.11648/j.ajsea.20130203.12

[15] V. B. Singh, P. K. Kapur, and Mashaallah Basirzadeh. "Open Source Software Reliability Growth Model by Considering Change-Point." BVICAM's International Journal of Information Technology 4.

[16] Zhang X, Pham H. A software cost model with warranty cost, error removal times and risk costs. IIE Trans 1998;30:1135-42

[17] Hossain SA, Dahiya RC. Estimating the parameters of a non homogeneous Poisson-process model for software reliability. IEEETrans Reliab 1993; 42:604-12.

[18] Pham H, Zhang X. NHPP software reliability and cost models with testing coverage. Eur J Oper Res 2003; 145:445-54

[19] Bai CG, Hu QP, Xie M, Ng SH. Software failure prediction based on a Markov Bayesian network model. J Syst Software 2005;74:275-82

[20] Pham H. Software reliability and cost models- perspectives, comparison, and practice. Eur J Oper Res 2003;149:475-89

[21] Jeske DR, Zhang X. Some successful approaches to software reliability modeling in industry. J Syst Software 2005;74:85-99

[22] Anjum Mohd., Md. Asraful Haque, Nesar Ahmad. Analysis and Ranking of Software Reliability Models Based on Weighted Criteria Value, I.J. Information Technology and Computer Science, 2013, 02, pp.114, DOI: 10.5815/ijitcs.2013.02.01

[23] N. Ahmad, M.G.M. Khan, and L.S. Rafi, "Analysis of an Inflection S-shaped Software Reliability Model Considering Log-logistic Testing-Effort and Imperfect Debugging", International Journal of Computer Science and Network Security, 2011, Vol. 11 (1), pp. $161-171$.

[24] N. Ahmad, M.G.M. Khan, and L.S. Rafi, "A Study of Testing-Effort Dependent Inflection S-Shaped Software Reliability Growth Models with Imperfect Debugging", International Journal of Quality and Reliability Management, 2010, Vol. 27 (1), pp. 89 - 110.

[25] P.K. Kapur, P.K. and S. Younes, "Modeling an imperfect debugging phenomenon in software reliability", Microelectronics and Reliability, 1996, Vol. 36, pp. 645-50.

[26] N. Ahmad, M.G.M. Khan, and L.S. Rafi, "Inflection S-shaped software reliability growth models with testing-effort functions," Proceedings of the VI International Symposium on Optimization and Statistics, Aligarh Muslim University, Aligarh, India, 29-31 December, 2008.

[27] N. Ahmad, M.U. Bokhari, S.M.K. Quadri, and M.G.M. Khan, "The exponentiated Weibull software reliability growth model with various testing-efforts and optimal release policy: a performance analysis", International Journal of Quality \& Reliability Management, 2008, Vol. 25 No. 2, pp. 211-35.

[28] N. Ahmad, M.G.M. Khan, and L.S. Rafi, "Software Reliability Modeling Incorporating Log-Logistic Testing-Effort with Imperfect Debugging”, in Proceedings of the International Conference on Modeling, Optimization and Computing (ICMOC-2010), Durgapur, India, Published by American Institute of 
Physics, 2010, pp. $651-657$.

[29] J. Iqbal, S.M.K. Quadri, and N. Ahmad, "Software Reliability Modeling with Learning-Factor Based Fault-Detection Rate", Proceedings of the 1st International Conference on Recent Trends in Computer Science \& Engineering" 8-9th February, 2014, Patna Bihar India (Narosa Publishing House)

[30] J. Iqbal, S.M.K. Quadri, and N. Ahmad, "An Imperfect- Debugging Model with Learning-Factor Based Fault-Detection Rate", Proceedings of the 8th International Conference on "Computing for Sustainable Global Development" (INDIACom-2014), Bharati Vidyapeeth's Institute of Computer Applications and Management (BVICAM), New Delhi (INDIA), 5th-7thMarch 2014.

\section{Authors' Profiles}

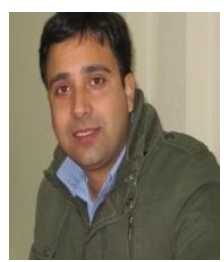

Javaid Iqbal received his B.Sc in Mathematics from the University of Kashmir, and Masters in Computer Applications from the same university in 2004. He completed his Ph.D from the University of Kashmir in 2014. His research interests are Software engineering, Software reliability engineering and reliability modeling, and mathematical modeling of dynamic systems. He is a member of ACM, CSI and IETE.

How to cite this paper: Javaid Iqbal,"Analysis of Some Software Reliability Growth Models with Learning Effects", International Journal of Mathematical Sciences and Computing(IJMSC), Vol.2, No.3, pp.58-70, 2016.DOI: $10.5815 / \mathrm{ijmsc} .2016 .03 .06$ 